\title{
Influence of mean distance between fibers on the effective gas thermal conductivity in highly porous fibrous materials
}

\author{
S.A.Lurie ${ }^{\mathrm{a}, \mathrm{b} *}$, Y.O. Solyaev ${ }^{\mathrm{a}, \mathrm{b}}$, D.V. Lizunovac, L.N. Rabinskiy ${ }^{\mathrm{c}}$, V.M. Bouznik ${ }^{\mathrm{d}}$, O. Menshykov ${ }^{\mathrm{e}}$ \\ anstitute of Applied Mechanics RAS, Moscow, Russia \\ ${ }^{b}$ Dorodnicyn Computing Center of RAS, Moscow, Russia \\ ${ }^{\mathrm{c}}$ Moscow Aviation Institute, Moscow, Russia \\ ${ }^{\mathrm{d}}$ All-Russian Research Institute of Aviation Materials Moscow, Russia \\ ${ }^{e}$ College of Physical Sciences, University of Aberdeen, Scotland, UK
}

\begin{abstract}
The paper considers the problem of definition of characteristic length parameter and Knudsen number in the calculation of the effective coefficient of thermal conductivity of highly porous fibrous materials. As the characteristic length it is proposed to use the parameter of average distance between fibers instead of the commonly used parameter of the average pore size. We introduced the technique for determining the average distance between fibers in the fibrous highly porous structures with different spatial orientation, volume content, diameter and length of fibers. The technique is based on the direct measurements of the distances between the fibers in representative volume elements (RVE) of fibrous structures. The requirements for the minimum size of the RVE are established. It is shown the convergence of the algorithm whenever we increase the number of measurements in various similar RVE of the same structure. We held the calculations of the average distance between the fibers in the three-dimensional isotropic structures with different volume content and diameter of the fibers. It is shown that the average distance between the fibers is always greater than the average pore size that determined on the basis of geometric methods or based on the known approximate analytical evaluations. It is proposed the modification of a simple analytical formula for the prediction of the average distance between the fibers in the highly porous structures. It is shown that the use of the parameter of average distance between fibers instead of the average pore size leads to the 2-3 times increase of the estimated gas effective thermal conductivity in the pores of the fibrous structure under low pressure.
\end{abstract}

Keywords: fibrous materials, characteristic length, Knudsen number, thermal conductivity, mean distance between fibers, pore size

\section{Introduction}

Fibrous high porous structures and materials are widely used in different applications, such as thermal insulation [1], filters [2], gas diffusion layers in fuel cells [3], sound-absorbing materials [4, 5], electromagnetic shielding [6], papers [7], textiles [8], biological systems [9, 10]. One of the most important characteristic that determines the transport properties of these materials is the average pore size or the average distance between fibers. This characteristic is decisive in the analytical assessment of permeability [11] and thermal conductivity of highly porous fibrous materials [1, 12]. Pore size is also important for the simulation of biological processes, for example, in tissue engineering [13] or in the modeling of cell migration through the fibrous structures [14].

The three-dimensional models and two-dimensional images of fibrous structures can be obtained using X-ray microtomography [15] or different microscopy techniques [16, 17 and references therein]. In the simulations, the models of fibrous structures could be generated using random algorithms with given structure parameters: volume content of porosity, lengths, diameters and

\footnotetext{
* Corresponding author, e-mail: salurie@mail.ru, address: 125040, Leningradsliy prosp., 7, Moscow, Russia
} 
orientations of the fibers and the characteristics of its tortuosity. The possibility to generate these models is implemented, for example, in systems Digimat (MSC) and GeoDict (Math2Market). The algorithms of generation are described in [18-21].

There are several basic methods for determining the mean pore size and the distribution of pore size in stochastic fibrous structures. The most common used methodology is the geometrical method for the pore size distribution determination $[22,23,17]$. This technique is based on the maximum packing algorithm of largest spheres in the pore space between fibers. Average size and size distribution of these spheres, respectively, are representative average size and distribution of pore size in the material. The modification of this approach is the porosimetry pore size distribution determination technique wherein the calculation takes into account only the spheres that form the connected space, and other spheres corresponding to isolated pores are excluded from consideration [22, 24]. Another widely used method for determining the average pore size in fibrous medias is capillary penetration simulation [20, 25, 26]. This methodology is based on the fluid flow simulations in micro-size RVE of fiber structures. According to the results of the simulation it is determined the kinetics of liquid penetration through the fibrous medium. Then, by comparing the obtained results and the known analytical expressions for the kinetics of liquid front in the parallel cylindrical channels, effective pore radius in a stochastic fiber structure is determined [20, 26]. In [26] on the basis of numerical simulation a comparison of different geometric and capillary penetration methods for determination of pore radius in fibrous structures was made. It was shown that predictions are strongly depend on the modeling techniques. Therefore, the choice of technique of average pore size prediction in the fiber structure must depend on the considered physical processes.

Note that for the planar structures it is known the analytical expressions to determine the average pore diameter through the diameter and volume content of fibers [18, 27, 28]. The study of the distribution of pore sizes in planar and multi-planar fibrous structures was carried out in [18, 29, 28 and references therein]. It has been shown that for such structures for in-plane pore diameters it is realized the gamma distribution, and for out-of-plane pores size it is realized the exponential distribution. Later, it was also found [30], that in such analytical models the pore size distribution could be underestimated, compared with the experimental data obtained on the basis of capillary flow porometry. For the threedimensional fiber networks on the base of a statistical analytical approach it was established [31] that in structures with extended fibers, irrespective of their isotropic or anisotropic orientation, it is realized the Rayleigh distribution of the pore diameters with the only one independent parameter ("nearest-obstacle distance"), which is linearly proportional to the average pore size.

In the simulations of highly porous fibrous thermal insulation it is often used the approximate analytical expression for the average pores size, that obtained from the model of ordered cylindrical fibers [1, 12, 32-34]:

$\delta=\xi \frac{d}{f}$

where $d$ is average diameter of fibers, $f$ is volume content of fibers, $\xi=\pi$ / 6 for three-dimensional structures and $\xi=\pi / 4$ for planar structures.

Expression (1) is used to determine the effective thermal conductivity of the gas in the pores through the Knudsen number during the calculation of the effective thermal conductivity of the fibrous medium [1, 12, 32-35] as follows:

$k=k_{s}+k_{g}+k_{r}$

$k_{g}=\frac{k_{g 0}}{1+2 \frac{\beta}{P r} K n}$

where $k$ is effective thermal conductivity of fibrous material; $k_{s}, k_{r}$ are solid and radiant parts of thermal conduction in fibrous material, the calculation of which is described, for example, in [1, 33]; $k_{g}$ is the effective gas thermal conductivity; $k_{g 0}$ is gas thermal conductivity at atmospheric pressure, $\beta=[2 \gamma /(\gamma+1)][(2-\alpha) / \alpha][21] ; \gamma=c_{p} / c_{v} ; \alpha=4 m_{g} m_{s} /\left(m_{g}+m_{s}\right)^{2} ; c_{p}, c_{v}$ are specific heats of 
gas at constant pressure and volume; $m_{g}, m_{s}$ are molecular weight of gas and solid phases; Knudsen number is defined as the ratio of molecular mean free path $\lambda$ and some characteristic length of considered structure $L_{c}: K n=\lambda / L_{c}$.

The molecular mean free path is determined by the formula [32, 36]:

$\lambda=\frac{k_{B} T}{\sqrt{2} \pi d_{m}^{2} P}$

where $k_{B}$ is the Boltzmann constant, $T$ is the temperature, $d_{m}$ is the gas collision diameter, $P$ is the pressure.

As a characteristic length $L_{c}$ in the study of heat transfer in fibrous medias, it is usually taken an average pore size, found by the formula (1), that is: $K n=\lambda / \delta[1,12,32-34]$. However, it is possible that in such calculations as characteristic length it is more appropriate to use the parameter of average distance between fibers $\delta^{*}$, which is obviously greater than the distance between the ordered mutually perpendicular cylinders or than geometrically defined average pore size (Fig. 1). That is, to determine the Knudsen number it is proposed to compare the molecular mean free path of the gas and the average distance between the fibers, assuming that the fibrous structure is unordered, and that molecules can move from any given point in any direction and not within a certain spherical pore of average size (Fig. 1). Determination of the average distance between the fibers of the three-dimensional stochastic structure requires a more complex geometrical approach, which is discussed in this article. We also note here that it is known that the values of average pore size found by formula (1) are 1.5-2 times lower compared to the values established experimentally [37, 12 and references therein]. Also note that in the simulations of heat transfer in fibrous media apart from the expression (1) for the approximate determining of average pore size, are also applied other estimates based, for example, on a comparison of pore volume in RVE with equal-size spherical volume, diameter of which is taken as the average pore size [38-40]. This method based on the assumption of equivalent spherical geometry of pores, is also used for porous media image processing [41].

The purpose of this work is the formulation of algorithm for direct calculation of average distance between fibers in the three-dimensional models of highly porous fibrous materials. We study the convergence of the algorithm for different values of the structural parameters. We make a comparison of the found values of the average distance between the fibers and the average pore diameter, which is determined by the previously known methods. We also presented the results of demonstrative calculations of the gas effective thermal conductivity in the highly porous fibrous material using the found values of the average distance between fibers.
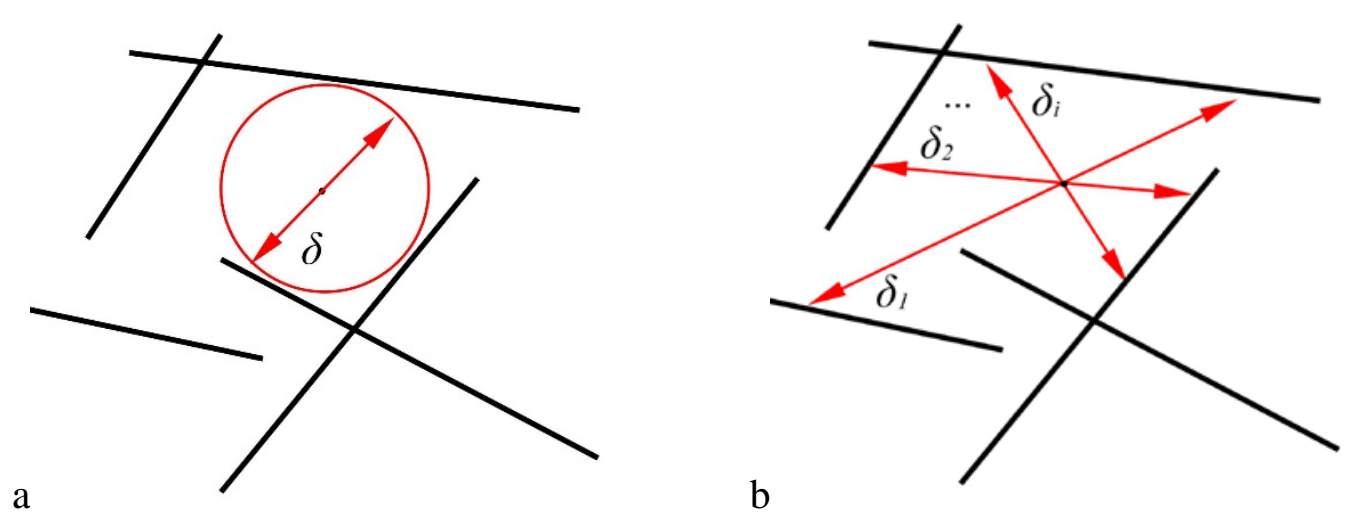

Fig. 1. Comparison of the pore size $\delta$, found using geometrical method based on the maximum packing algorithm of largest spheres in the pore space of the fibrous structure (a), and the

parameter of average distance between the fibers $\delta^{*}$, determined on the basis of direct measurements (b). The average pore size is always smaller than the average distance between 
fibers: $\delta<\delta^{*}=\frac{1}{N} \sum_{i=1}^{N} \delta_{i}$ ( $N$ is number of measurements). For clarity, it is shown a planar fiber structure, a similar relation holds in the three-dimensional structure.

\section{Methodology}

In the following simulations the realistic three-dimensional RVE of fibrous materials were generated using the system Digimat-FE (MSC). The beam-type inclusions are used as the model of the fibers. To construct the RVE of fibrous material in Digimat-FE system it is necessary to set the volume content of the fibers, their diameter, length and orientation. It must be selected the size and shape of a representative fragment. In subsequent calculations we'll use only the fragments of cubic form and consider only straight fibers. Note that the system can generate RVE, containing fibers of different materials with different diameters and with a predetermined length distribution. The test calculations consider only materials with a fixed diameter and length of fibers, suggesting that these characteristics are average for the given material structure. The orientation of the fibers will be specified using tensor [21]. As a result of Digimat-FE system work it is created the RVE which satisfies the introduced requirements imposed to the structure parameters (volume content, fiber orientation, etc.). Location and direction of each fiber are determined by the coordinates of its center and by three angles. For each fiber it is known its length and diameter. An example of generated RVE is shown in Fig. 2.
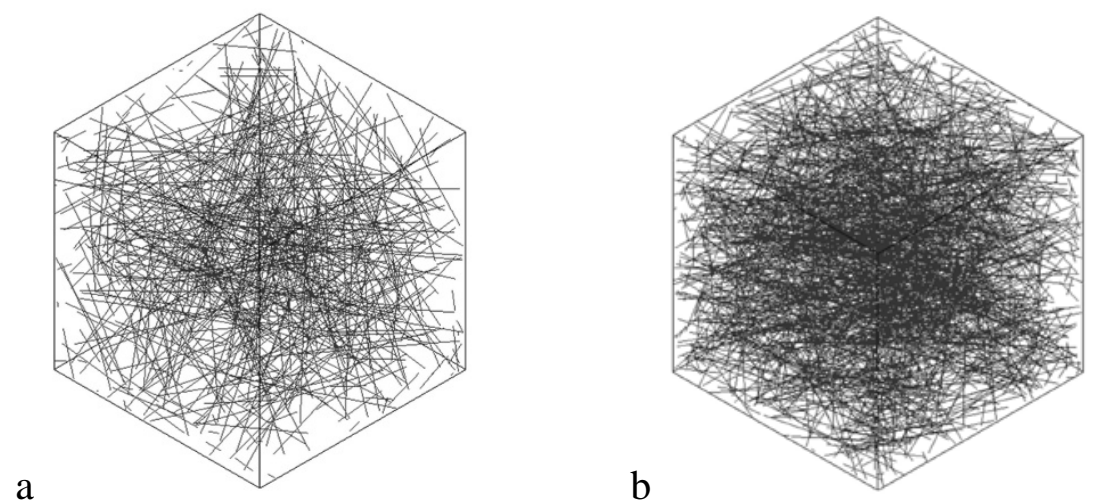

Fig. 2. Examples of RVE of fibrous structures generated in Digimat-FE system with the use of straight beams inclusions of $1 \mu \mathrm{m}$ in diameter and $10 \mu \mathrm{m}$ long and with $10 \%$ of volume content, a: isotropic structure, RVE size of $50 \mu \mathrm{m}$, b: an anisotropic structure with an average angle of $80^{\circ}$ of the fibers to the vertical direction, RVE size is $80 \mu \mathrm{m}$. Fibers are modeled as straight lines that are axes of real fibers-cylinders.

To analyze the geometry of established RVE, we import obtained data to the Wolfram Mathematica system. As a result, we have saved dataset, which contains the numbers of $n$ generated fibers, coordinates of their centers $\mathbf{r}_{i 0}=\left(x_{i 0}, y_{i 0}, z_{i 0}\right),(i=\overline{1, n})$, values of direction angles $\theta_{i}$ and $\varphi_{i}$, lengths $l_{i}$ and diameters of fibers $d_{i}$. Next we provide the processing of RVE geometry to estimate the average distance $\delta^{*}$ between the fibers. For that it is introduced a standard parametrization of the line segments set $\ell_{i} \in \mathfrak{L}$ that simulate fibers, as follows:

$\mathfrak{L}=\left\{\ell_{i}: \mathbf{r}_{i}=\mathbf{r}_{i 0}+t \mathbf{p}_{i}, t \in T_{i}=\left[t_{i}^{\min }, t_{i}^{\max }\right], i=\overline{1, n}\right\}$

Here $\mathbf{r}_{i}=\mathbf{r}_{i}(t)$ is the radius-vector of points belonging to the line segment $\ell_{i} ; \mathbf{p}_{i}=\left(a_{i}, b_{i}, c_{i}\right)$ is a vector determining its orientation with components $a_{i}=\sin \theta_{i} \cos \varphi_{i}, b_{i}=\sin \theta_{i} \sin \varphi_{i}, c_{i}=\cos \theta_{i}$; $t$ is a parameter that varies between $T_{i}=\left[t_{i}^{\min }, t_{i}^{\max }\right]$ so that the ends of the fiber-segment do not extend beyond the RVE. 
Note that after the importing of the data from Digimat-FE system, we do not know the values $t_{i}^{\min }$ and $t_{i}^{\max }$, and the coordinates of centers of fibers-segments $\mathbf{r}_{i 0}$ can lie outside of representative fragment if it is smaller than the length of the fiber or if the fibers lie close to its borders. Therefore, for further calculations the range of variation of the parameter $t$ for each segment $\ell_{i} \in \mathfrak{L}$ is determined with consideration of restrictions on the length of the fiber and the size of RVE:

$$
\left\{\begin{array}{l}
t_{i}^{\min }=\min _{\mathbf{r}_{i} \in \mathscr{D}}(t), \\
t_{i}^{\max }=\max _{\mathbf{r}_{i} \in \mathcal{D}}(t), \\
\left|\mathbf{r}_{i}-\mathbf{r}_{i 0}\right|=|t| \leq l_{i} / 2, \\
\mathbf{r}_{i}=\mathbf{r}_{i 0}+t \mathbf{p}_{i}, \quad i=\overline{1, n .} .
\end{array}\right.
$$

Here $\mathscr{D}=\{(x, y, z): x \in[0, D], y \in[0, D], z \in[0, D]\}$ is a cubic area which is occupied by a RVE.

Next, we could determine the average distance between the fibers. The exact value of $\delta^{*}$ in the considered RVE can be found as follows:

$$
\delta^{*}=\frac{1}{2 \pi^{2} L} \int_{\mathfrak{L}}\left(\int_{0}^{\pi} \int_{0}^{2 \pi} \delta_{\min }\left(t, \ell_{i}, \varphi, \theta\right) d \varphi d \theta\right) d t,
$$

where the external integration is carried out along all the segments of $\mathfrak{L}$, and besides, along each segment $\ell_{i} \in \mathfrak{L}$ the parameter $t$ varies within the limits defined by the expression (2); internal integration is carried out in all possible directions $(\varphi, \theta)$ of the fixed point of the segment $\ell_{i}$, except for the direction of the segment itself $\varphi=\varphi_{i}$ and $\theta=\theta_{i} ; \delta_{\min }\left(t, \ell_{i}, \varphi, \theta\right)$ is the distance from the point with coordinate $t$, which lies on a segment $\ell_{i}$, to the other closest segment-fiber, located in the direction $(\varphi, \theta) ; L=\sum_{i=1}^{n} l_{i}$ is the total length of fibers-segments included in the RVE.

Thus, in the expression (6) it is determined the distance from each point of each fiber to the other closest fiber in all directions with respect to the considered point, and then the average value of all the distances is found. The exact definition of $\delta^{*}$ using the expression (6) is associated with significant computational difficulties, since the length of the path of integration in the external contour integral can be several orders greater than the size of a RVE. Any analytical or approximate estimations of this value, with the exception of (1), as far as we know, doesn't exist.

In this paper we propose to use a faster statistical algorithm. For this we consider a set of points $\mathcal{P}$ in the pores in the center of a representative fragment (Fig 2a.):

$\mathscr{P}=\left\{P_{i}=\left(P_{x i}, P_{y i}, P_{z i}\right): P_{i} \in \mathscr{D}^{\prime}, P_{i} \notin \mathfrak{L}, i=\overline{1, N_{p}}\right\}$,

Here $\mathscr{D}^{\prime}$ is a central region of a representative fragment, which in further test calculations is selected as follows: $\mathscr{D}^{\prime}=\{(x, y, z): x \in[0.45 D, 0.55 D], y \in[0.45 D, 0.55 D], z \in[0.45 D, 0.55 D]\}, \quad N_{p} \quad$ is a number of selected points.

Next, choose a set of vectors $\mathcal{Q}=\left\{\mathbf{q}_{i}\right\}$, in a direction of which we will evaluate the distance from the fixed selected point $P_{i}$ to the nearest fiber. We define these directions as follows:

$$
\mathcal{Q}=\left\{\mathbf{q}_{i}=\left(q_{x i}, q_{y i}, q_{z i}\right): q_{x i}=\sin \theta \cos \varphi, q_{y i}=\sin \theta \sin \varphi, q_{z i}=\cos \theta, \theta \in[0, \pi], \varphi \in[0, \pi], i=\overline{1, N_{q}}\right\}
$$

Here $N_{q}$ is a number of selected directions defined by two angles $\theta$ and $\varphi$. The limits of variation of these angles are chosen taking into account the fact that in further calculations simultaneously with the selected directions $\mathbf{q}_{i}$ the directions $-\mathbf{q}_{i}$ also will be considered.

The coordinates of the points $\mathcal{P}$ and the components of the vectors $\mathcal{Q}$ are selected using a random number generator within a predetermined range of acceptable values. Then through every point of $\mathcal{P}$ 
it is built a set of auxiliary measuring lines $\mathfrak{L}_{P}$, which directions are determined by the vectors from $Q$ (Figure 2b.):

$$
\mathfrak{L}_{P}=\left\{\ell_{i j}: \mathbf{r}_{i j}=P_{i}+s \mathbf{q}_{j}, s \in S_{i j}, i=\overline{1, N}_{p}, j={\overline{1, N_{q}}}_{\cdot}\right\}
$$

Here $S_{i j}=S\left(P_{i}, \mathbf{q}_{j}\right)=\left[\mathrm{s}_{i j}^{\min }, \mathrm{s}_{i j}^{\max }\right]$ is the range of acceptable values of the parameter $s$, which depends on the point under consideration $P_{i}$ and direction $\mathbf{q}_{j}$, and varies so that the boundary points of segment $\ell_{i j}$ belong to the faces of the cubic area $\mathscr{D}$, occupied by a representative fragment. Thus, the values $\mathrm{s}_{i j}^{\min }$ and $\mathrm{s}_{i j}^{\max }$ are found by solving the problem:

$$
\left\{\begin{array}{l}
s_{i j}^{\min }=\min _{\mathbf{r}_{i j} \in \mathscr{D} .}(s) \\
s_{i j}^{\max }=\max _{\mathbf{r}_{i j} \in \mathcal{D} \text {. }}(s) \\
\mathbf{r}_{i j}=P_{i}+s \mathbf{q}_{j}, i=\overline{1, N}_{p}, j=\overline{1, N_{q}} .
\end{array}\right.
$$

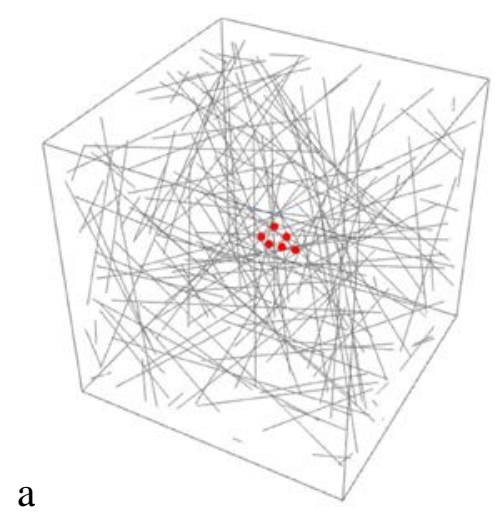

b
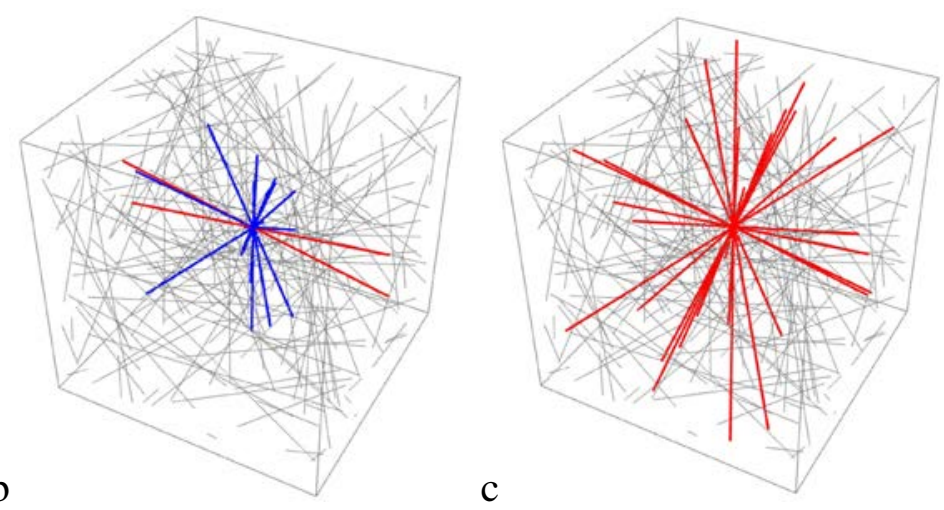

Fig. 3. Method for determination of the average distance between fibers in a highly porous fibrous structures. a: Set of points $\mathcal{P}$ selected in the central region of the pore space of RVE, b: The directions in which the distance between fibers will be measured and the corresponding built auxiliary measuring lines $\ell_{i j}$. Such directions and segments are constructed at each selected point of $\mathcal{P}$, c: Found distances between the fibers in selected directions (blue lines) and directions that were not covered with fibers (red line). The presence of such uncovered directions shows the need of the RVE size increasing.

Construction of the segments $\ell_{i j}$ is sub-procedure that is necessary to measure the distance from the point $P_{i}$ to the nearest to it fiber along the direction $\mathbf{q}_{j}$. To do this, firstly, let's determine the minimum distances from each fixed measuring line $\ell_{i j} \in \mathfrak{L}_{P}$ to each fiber $\ell_{k} \in \mathfrak{L}$ in the RVE. To do this let's solve the following problems:

$$
\left\{\begin{array}{l}
\left(\delta_{i j k}\right)_{\min }=\min _{t \in T_{k}, s \in S_{i j}}\left(\delta_{i j k}(t, s)\right), \\
\delta_{i j k}(t, s)=\left(\left(x_{k 0}+t a_{k}-P_{x i}-s q_{x j}\right)^{2}+\left(y_{k 0}+t b_{k}-P_{y i}-s q_{y j}\right)^{2}+\left(z_{k 0}+t c_{k}-P_{z i}-s q_{z j}\right)^{2}\right)^{0.5}, \\
i=\overline{1, N}_{p}, \quad j=\overline{1, N_{q}}, \quad k=\overline{1, n} .
\end{array}\right.
$$

For each fixed segment $\ell_{i j}$ let's determine which fibers $\ell_{k}$ are closer to it than to the value of its own radius $d_{k} / 2$ (intersect with it), that is, we select the following subsets $\mathfrak{L}_{i j} \subset \mathfrak{L}$ :

$\mathfrak{S}_{i j}=\left\{\ell_{k} \in \mathfrak{L}:\left(\delta_{i j k}\right)_{\min } \leq d_{k} / 2\right\}$, 
wherein we determine the points $P_{i j k}$, in which there is an intersection of measuring line $\ell_{i j}$ and the line-fiber $\ell_{k}$. At these points, the distances between this lines reach the minimum and it does not exceed the diameter of the fibers. Location of points $P_{i j k}$ is defined by a value of parameter $s=s_{i j k}^{*}$ in parameterization formulas (7) for the segments $\ell_{i j}$.

Further, in each subset $\mathfrak{L}_{i j}$ we need to select the two fibers, which are located closest to the point $P_{i}$ it the directions $\mathbf{q}_{j}$ and $-\mathbf{q}_{j}$. In fact, we find here the two fibers that are "visible" from a given point $P_{i}$ in the predetermined directions $\mathbf{q}_{j}$ and $-\mathbf{q}_{j}$. Let's denote the found closest fibers as $\ell_{i j k}^{+}$and $\ell_{i j k}^{-}$. Parameters defining the location of the contact points of segment-meter $\ell_{i j}$ with segments $\ell_{i j k}^{+}$and $\ell_{i j k}^{-}$, let's denote as $s=s_{i j k}^{+}$and $s=s_{i j k}^{-}$, respectively. Then we can write:

$\ell_{i j k}^{+} \in \mathfrak{L}_{i j}: S_{i j k}^{+}=\min _{k=1, n}\left(s_{i j k}^{*}\right)>0$,

$\ell_{i j k}^{-} \in \mathfrak{S}_{i j}: s_{i j k}^{-}=\max _{k=1, n}\left(s_{i j k}^{*}\right)<0$.

The desired distance between closest fibers at a given point $P_{i}$ in the direction $\pm \mathbf{q}_{j}$ is given by the sum:

$\delta\left(P_{i}, \mathbf{q}_{j}\right)=s_{i j k}^{+}+\left|s_{i j k}^{-}\right|$

Thus we find the distance between the fibers at all the selected points $P_{i}$ in all directions $\mathbf{q}_{j}$ (Fig. 2c). Through the analysis of sets of found values, we obtain the statistical distribution of the distances between the fibers. The average distance between the fibers in this RVE is given by:

$\delta_{R V E}=\frac{1}{N_{P} N_{q}} \sum_{i=1}^{N_{P}} \sum_{j=1}^{N_{q}} \delta\left(P_{i}, \mathbf{q}_{j}\right)$.

It is obvious that, for sufficiently large $N_{p}$ and $N_{q}$, the value determined by the expression (8) should coincide with the exact value (6) which can be calculated for a particular RVE.

$\delta^{*}=\frac{1}{N_{R V E}} \sum_{k=1}^{N_{R V E}}\left(\delta_{R V E}\right)_{k}$

In the proposed algorithm we can immediately specify the requirement to the RVE size of the fiber structure. The RVE should be large enough so that all the points $P_{i j k}$ could be found inside it, i.e. the fibers included in the RVE should lie over all directions relative to the selected points $P_{i}$ (Fig. 2c). It follows that if the material is highly porous and volume content of fibers is low, then for the calculations we need to considered larger RVE's. A detailed study of this problem is given below. Also it should be taken into account that for a reliable predictions of the structure characteristics, it's necessary to consider several similar RVE's generated using random algorithms, and to find an average value:

$\delta^{*}=\frac{1}{N_{R V E}} \sum_{k=1}^{N_{R V E}}\left(\delta_{R V E}\right)_{k}$

Here $N_{R V E}$ is the number of considered RVE's, and $\left(\delta_{R V E}\right)_{k}$ is found average distance between the fibers in the $k$-th fragment. The total number of measurements on different representative fragments is $N=N_{R V E} N_{p} N_{q}$.

The proposed algorithm of the mean distance between fibers determination is shown in the Figure 3. 


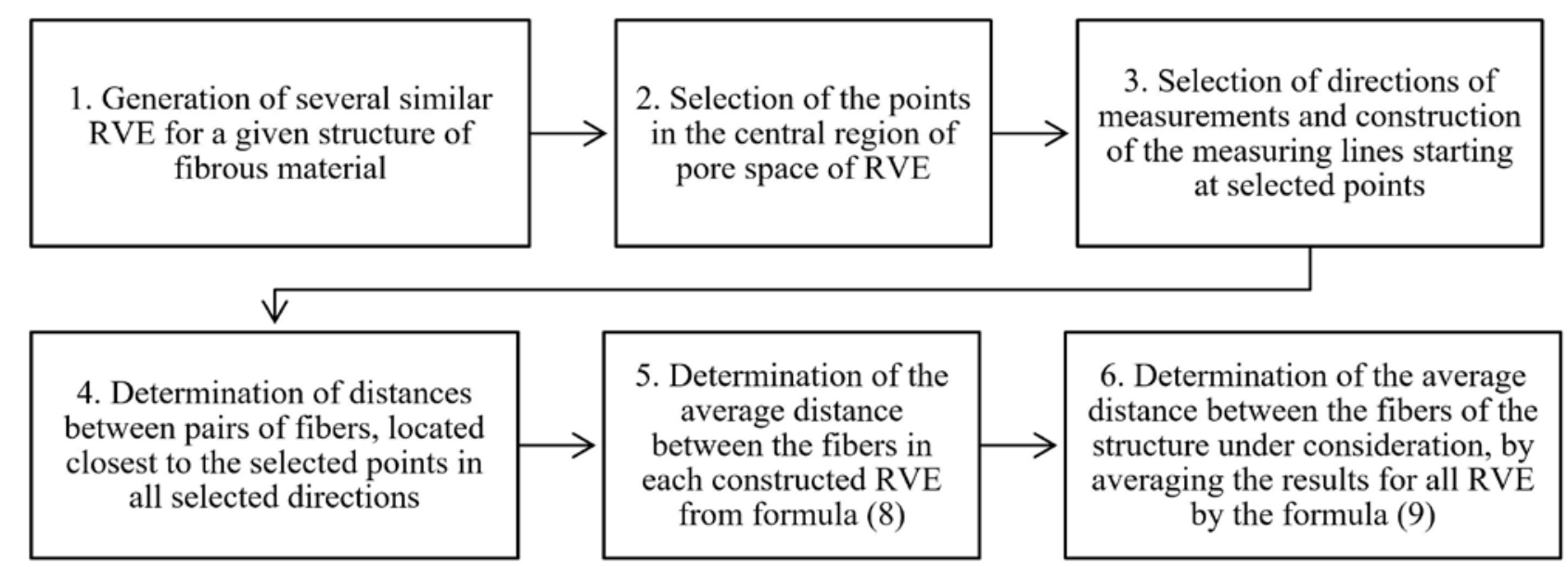

Fig. 4. The algorithm for determining the average distance between fibers in a highly porous fibrous structures

\section{Results and discussion}

\subsection{Minimum RVE size and convergence of the algorithm}

First and foremost, we must determine the minimum adequate size of representative fragment $D$ and the minimum number of measurements of the distance between the fibers $N$, which ensure the accuracy and reliability of the calculations. To determine the average distance between the fibers, it is necessary that a representative fragment was large enough to contain a lot of fibers, creating a dense covering of the central region of fragment in which we carry out the measurements. This is necessary to be able to measure the distance between the fibers at all selected test points and directions. If at least in one direction the measuring line does not overlap with any of the fibers in the RVE, this means that in this structure the distance between the fibers can exceed the size of the RVE and it's necessary to increase it. Otherwise we will get underestimated values for the desired averaged values. Figure 5 shows how many uncovered directions occur in RVE's of different size with a volume content of fibers of $10 \%$ and $15 \%$. During the calculations at the center of the fragments we chose three test points $N_{P}=3$, and the number of measurement directions was $N_{q}=40$, that is, in fact, the measurement of the distances between the fibers was conducted 120 times in each RVE. By increasing the RVE size, the number of uncovered directions becomes smaller (Fig. 4), as the larger RVE contains more fibers, and it becomes less transparent (see. Fig. 2a, b). When the size of RVE is more than $60 \mu \mathrm{m}$ such uncovered directions in these structures hardly occur (few number of uncovered directions have no significant impact on the value $\delta$ ). The central region of the fragment is completely covered by fibers. Therefore, we can assume that for the considered structures this size of RVE is the minimum. Figure 6 shows the found values of minimum sizes of RVE for the structures with different volume content and different diameter of fibers. When the diameter of the fibers is increasing it is necessary to increase the size of RVE, while increasing their volume content, RVE size can be taken smaller (Fig. 6). For all the structures considered above, the condition that RVE of the minimum allowed size must contain approximately 300-500 fibers is satisfied. These fibers do not fall entirely within a RVE, and can be dissected into smaller pieces to accommodate in it. The number of these small parts in the fragment may be 1200-1400. Thus in Digimat system it is modeled stochastic structure, which includes not only the entire fibers, but fiber ends lying in the neighboring regions out of a space of considered RVE. It should be noted that there is no need to consider RVE larger in a relation of the minimum found values (Fig. 6). Increasing of RVE doesn't lead, firstly, to the appearance of the uncovered directions, and secondly, does not change the calculated values of the average distance between fibers. It is quite evident result from the fact that RVE generation algorithms work identically on fragments of various sizes. 


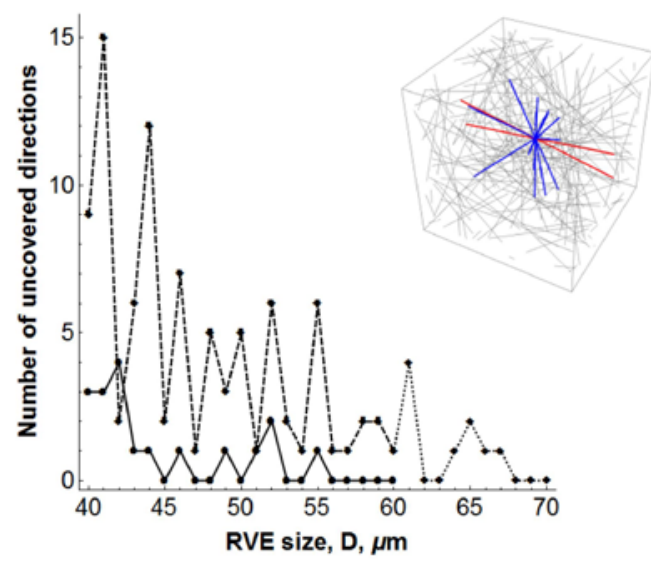

Fig. 5. The number of uncovered directions during the measurements in the RVE of different size with fibers contain of 15\% (solid line) and 10\% (dashed line). The fiber diameter is $1 \mu \mathrm{m}$, length -

$100 \mu \mathrm{m}$, orientation - isotropic. The existence of uncovered directions means that the distance between the fibers in these directions exceeds the size of the RVE. For the structure under consideration the minimum RVE size is $60 \mu \mathrm{m}$ for $15 \%$ of the fiber content and $70 \mu \mathrm{m}$ for $10 \%$. In these fragments the fibers do not fit entirely, however, the average distance between them can be already determined, since uncovered directions in the center of fragment are lacking.

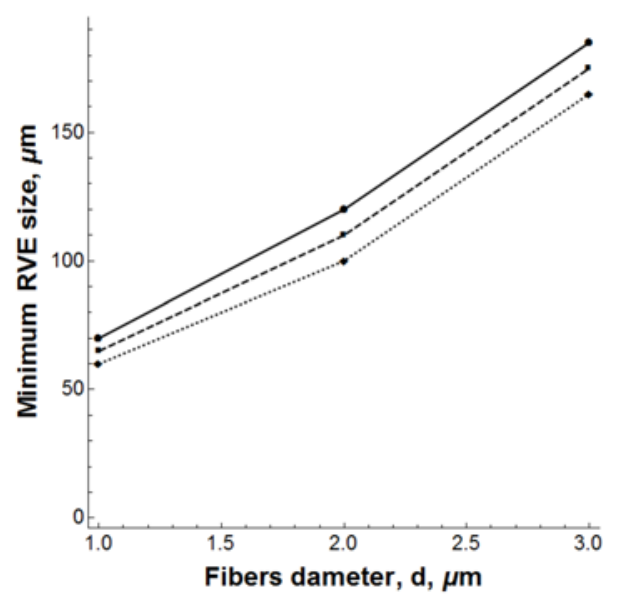

Fig. 6. The minimum required size of the RVE for fibrous structures with different diameters and volume content of fibers (solid line $-10 \%$, dashed line $-12.5 \%$, dotted line $-15 \%$ ). Fiber length is $100 \mu \mathrm{m}$, orientation - isotropic. The minimum sizes of RVE are identified as a result of a series of calculations with the gradual increase of RVE for each type of structure. In a minimum fragment, there is no uncovered directions. This ensures the accuracy of the calculations, since in this case all the measured distances between the fibers do not exceed the size of RVE.

After the minimum size of RVE was found for the considered structure, it's necessary to check the convergence of the algorithm $\delta^{*}$ calculation when the number of measurements is increasing. It is also necessary to determine how many repeated measurements we need to conduct to reliably calculate the value $\delta^{*}$. In fact, we have to consider a lot of RVE of the same type and carry within them a lot of measurements, such that the further increasing of the measurements number doesn't change the determined averaged value $\delta^{*}$. Figure 7 shows the convergence of the presented algorithm. Here it is shown that by increasing the number of measurements, the desired value of the average distance between fibers goes to some asymptotic value. Calculations are made for isotropic structure formed by fibers of $100 \mu \mathrm{m}$ of length with different diameters and volume content. For each type of structure from 1 to 10 of RVE have been reviewed, and at each fragment there were chosen three points in which the measurements were carried out in 10 directions. I.e. $N_{R V E}=1 \ldots 10, N_{p}=3$, 
$N_{q}=10, N=N_{R V E} N_{p} N_{q}=30 \ldots 300$. Note that the calculations were quite intensive, since it were carried out on the fragments with the size more than $60 \mu \mathrm{m}$, containing 1200-1500 parts of fibers.

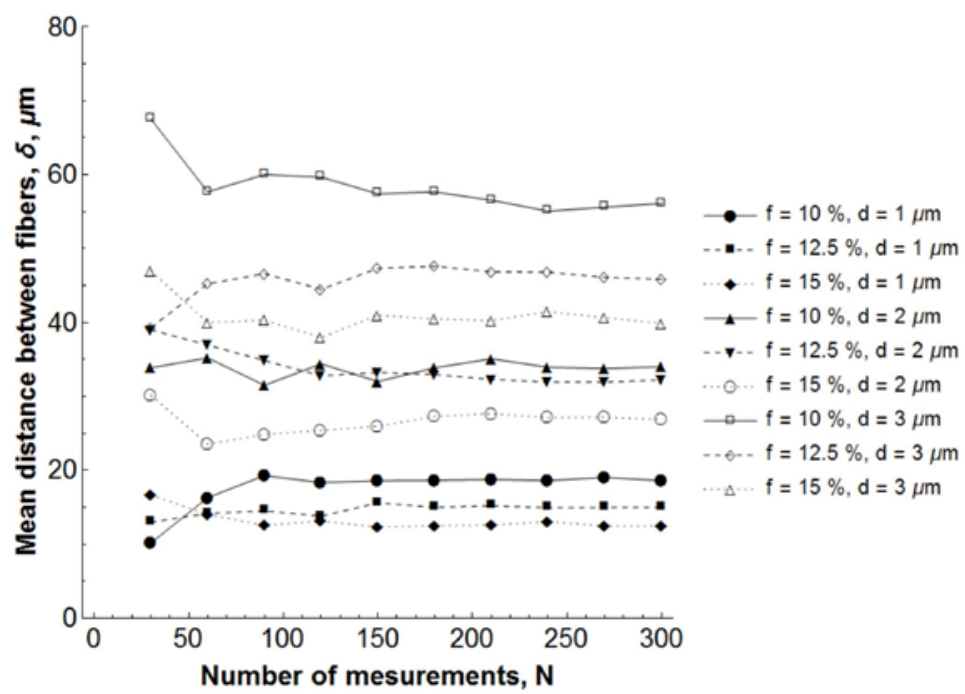

Fig. 7. Convergence of the algorithm for determining the average distance between the fibers $\delta^{*}$ during increasing the number $N$ of measurements in structures with different fibers diameters and volume contents. Fiber length is $100 \mu \mathrm{m}$. When the number of measurements is increasing, the required value of $\delta^{*}$ goes to the asymptote. The calculations considered from 1 to 10 of RVE's of the same type for each type of structure $N_{R V E}=1 \ldots 10$. At each RVE we considered three points, and at each point the measurements were conducted in ten directions ( $N_{p}=3, N_{q}=10$ ), therefore the total number of measurements is $N=N_{R V E} N_{p} N_{q}=30 \ldots 300$.

Figure 8 shows the typical distribution of the distances between fibers for the structure, containing $12.5 \%$ of the fibers $100 \mu \mathrm{m}$ long and $1 \mu \mathrm{m}$ in diameter. This distribution is shown graphically as a found set of measuring lines, showing the distance between the fibers, and also as a probability histogram. The total amount of measurements in this fragment was $N=300$. It is shown that the real shape of the pore space in the fibrous stochastic structure may have a very complex configuration.
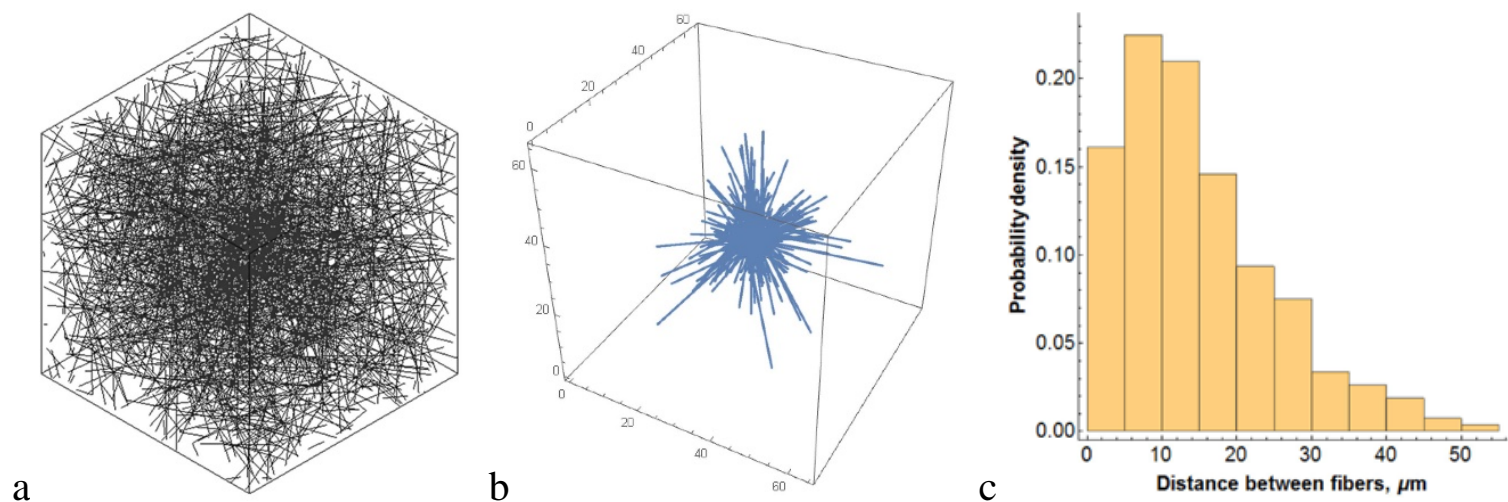

Puc. 8. Results of the mean distance between fibers estimation in the RVE which contains $12.5 \%$ of fibers with length $100 \mu \mathrm{m}$ and diameter $1 \mu \mathrm{m}$ (a). RVE size is $65 \mu \mathrm{m}$. Fibers are modeled as straight

lines (axes of real fibers-cylinders). Distribution of distances between fibers presented in the graphical form as the set of measuring lines between nearest fibers in different directions (b) and in the form of probability histogram (c). Total number of measurements is $N=300\left(N_{R V E}=10, N_{p}=3\right.$

$$
\text { , } \left.N_{q}=10\right) \text {. }
$$




\subsection{Comparison of the average distance between the fibers and the average pore size in fibrous structures}

Figure 9 shows a comparison of the average distance between the fibers and the average pore size. The calculation was performed for an isotropic structure containing fibers of a length of $100 \mu \mathrm{m}$ with different diameter and different volume content. The average pore size is determined on the base of a standard geometric algorithm [17, 22, 23] implemented in the GeoDict software and on the basis of the approximate formula (1), with the use of value $\xi=\pi / 6$. Calculation of the average distance between the fibers was held on the basis of the presented algorithm. The found values $\delta^{*}$, in fact, are asymptotic values to which the results of averaging for a large number of measurements are going (Fig. 7). It is evident that the obtained values of the average distance $\delta^{*}$ are approximately 3.5 times larger than the average pore size $\delta$. Therefore, in Figure 9 it is suggested an approximation (red lines) of the obtained results for $\delta^{*}$ by formula (1), but with another value of the coefficient of proportionality: $\delta^{*}=\xi d / f$, where $\xi=3 \pi / 5$. This approximation is quite a good description of the obtained statistical results for $\delta^{*}$. Exactly this approximation is proposed to use for the calculation of Knudsen number in the formula (2) for gas thermal conductivity in the fibrous materials with an isotropic fiber orientation.

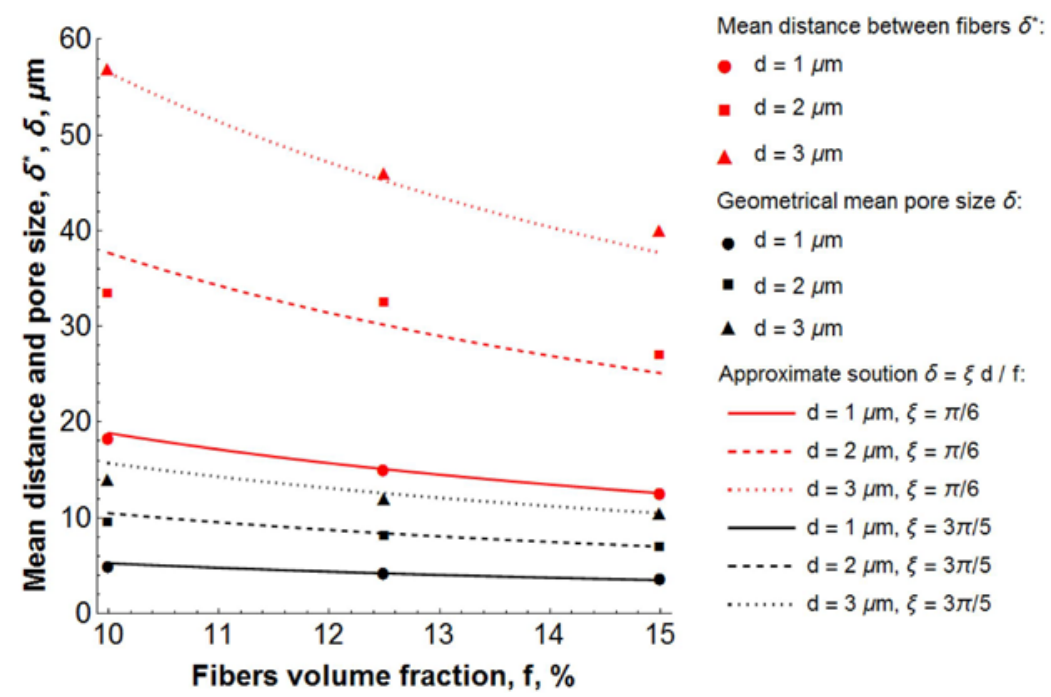

Fig. 9. Comparison of the found average distance between the fibers $\delta^{*}$ and the average pore size $\delta$ for an isotropic structure, formed by fibers of $100 \mu \mathrm{m}$ in length.

The average distance $\delta^{*}$ (red dots) is determined on the basis of the proposed algorithm. The average pore size is determined on the basis of known geometrical method. The solid lines show the predictions of approximate analytical solution $\delta=\xi d / f$. The average pore size is calculated by this formula for $\xi=\pi / 6$ (black lines). The average distance between the fibers is possible to be accurately predicted when $\xi=3 \pi / 5$ (red lines).

\subsection{Determination of the effective gas thermal conductivity using parameter of mean distance between fibers}

Figure 10 shows the impact of the characteristic length parameter on the effective thermal conductivity of air under various pressure. The calculations used the expression (2) and (3). The parameters for the air were taken from [42]. From these dependences it is seen that the specification of characteristic length value, for example, in 4 times, may alter the effective thermal conductivity of the gas in 2-3 times under low pressure conditions. Figure 11 shows the dependence of the effective thermal conductivity of the gas from pressure and temperature. Here, as the characteristic length we used the parameters of average size of pores $\delta(1)$ and the average distance between fibers $\delta^{*}$. It is shown that under low pressure the calculated effective thermal conductivity of the gas can be 2-3 
times higher, if the Knudsen number is calculated using the average distance between the fibers $\delta^{*}$ found by the proposed algorithm. If we consider that gas-phase thermal conductivity in fibrous materials may provide $80 \%$ (at $300{ }^{\circ} \mathrm{K}$ ) and about $10-50 \%$ (at $1000{ }^{\circ} \mathrm{K}$ ) of the total thermal conductivity [33, 43-46], then the received clarifications in certain ranges of temperature and pressure could be substantial. From the presented results, it follows that the contribution of the gas thermal conductivity may be higher, compared with previous estimates. Consequently, the values of parameters of radiative heat transfer models, which generally require the most complex identification methods [38, 47, 48], may be adjusted.

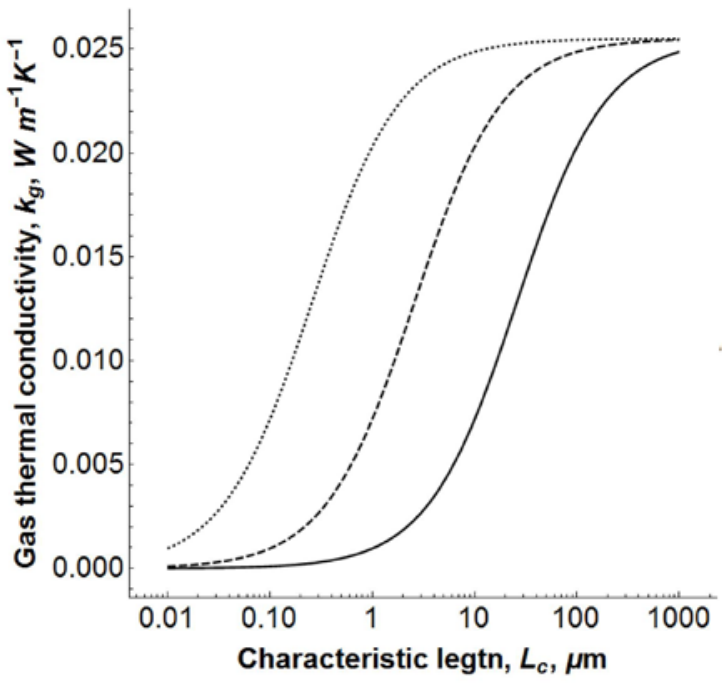

Fig. 10. Dependence of the effective thermal conductivity of air in the pores of the fiber media from the parameter of characteristic length $L_{c}$ under different pressures. The calculation is made by the analytical formulas (2) and (3), temperature is $300{ }^{\circ} \mathrm{K}$, pressure is $100 \mathrm{KPa}$ (solid line), $10 \mathrm{KPa}$ (dashed line), $1 \mathrm{KPa}$ (dotted line), parameters for air are taken from [42]. Clarification of value $L_{c}$ can lead to substantial amendments for the effective thermal conductivity of gas.

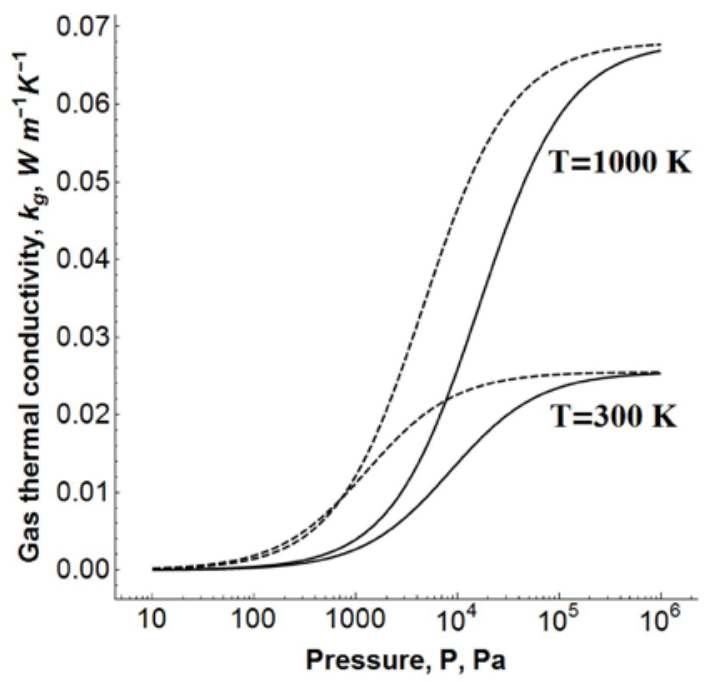

Fig. 11. Dependence of the effective thermal conductivity of air in the pores of the fiber medium from pressure using as the characteristic length the average pore size ( $L_{c}=\delta=5.2 \mu \mathrm{m}$, solid line) or the average distance between fibers ( $L_{c}=\delta^{*}=18.5 \mu \mathrm{m}$, dashed line). The material contains $10 \%$ of $1 \mu \mathrm{m}$ diameter fibers. Calculation are made according to the formulas (2) and (3), the temperature is $300{ }^{\circ} \mathrm{K}$, and $1000{ }^{\circ} \mathrm{K}$, the parameters for the air are taken from [42]. Formula (1) was used to assess

the average pore size. The average distance between the fibers was found on the basis of the proposed algorithm (see. Fig. 9). It is shown that for the considered conditions the use of parameter 
of the average distance between the fibers $\delta^{*}$ instead of the average pore size $\delta$ may give amendments of 2-3 times for the thermal conductivity of the gas under low pressure.

\section{Conclusions}

In this paper we proposed a new algorithm to determine the characteristic size of the pore space in the fibrous materials based on direct measurement of the statistically-average distance between fibers. We showed the convergence of the algorithm and specified the requirements for the minimum size of RVE. It is shown that the parameter of the average distance between fibers is always higher compared to the average pore size value found on the basis of geometric or approximate methods. It is proposed to use the value of the average distance between the fibers to calculate Knudsen number in the estimations of the gas thermal conductivity of fibrous structures. Wherein it is possible to use the simple solution $\delta^{*}=(5 \pi / 3) d / f$ for an approximate estimation of the average distance between the fibers in isotropic structures.

The proposed algorithm can be easily used in conjunction with standard parallel computing algorithms. It can be accelerated, for example by prior separation of all fibers into groups lying in the specified quadrants with respect to the middle region of a representative fragment. One can also speed up the algorithm by using vertex-representation for geometry of fragments. In such approach, we should not solve analytical problems to search the minimum distance, but we could define it by stepping extension of measuring lines from the selected point in the center of the fragment to the contact with the nearest fiber.

In the further work, it is necessary to explore the influence of the orientation, turtousity and different distribution of the size of the fibers. The analytical models predict the independence of the character of pore size distribution when orientation of the fibers is changing $[18,31]$ however it is predicted the change of average pore size - in the three-dimensional structures, it must be smaller, compared with flat and multi-layered structures (3). Therefore, on the base of the proposed approach we could try to obtain more accurate estimates of these values.

Presented results may be important in assessing of the gas-phase impact on the effective thermal conductivity of fibrous porous materials. Updates could be obtained by processing experimental data and by solving inverse problems for identification of the parameters of various models of radiativeconductive heat transfer in highly porous fibrous medias.

This work was supported by the Russian Science Foundation (grant number 14-33-00032).

\section{References}

[1] K. Daryabeigi, G.R. Cunnington, J.R. Knutson, Combined heat transfer in high-porosity high-temperature fibrous insulation: Theory and experimental validation, Journal of Thermophysics and Heat Transfer 25 (4) (2011) 536-546.

[2] X. Zhang, S. Cheng, X. Huang, B. E. Logan, The use of nylon and glass fiber filter separators with different pore sizes in air-cathode single-chamber microbial fuel cells, Energy Environ. Sci. 3 (5) (2010) 659-664.

[3] J.T. Gostick, Random Pore Network Modeling of Fibrous PEMFC Gas Diffusion Media Using Voronoi and Delaunay Tessellations, Journal of the Electrochemical Society 160 (8) (2013) F731-F743.

[4] S. Liu, W. Chen, Y. Zhang, Design optimization of porous fibrous material for maximizing absorption of sounds under set frequency bands, Applied Acoustics 76 (2014) 319-328.

[5] R. Fangueiro, Fibrous and composite materials for civil engineering applications, Elsevier 2011.

[6] Z.M. Huang, Y.Z. Zhang, M. Kotaki, S. Ramakrishna, A review on polymer nanofibers by electrospinning and their applications in nanocomposites, Composites Science and Technology 63 (15) (2003) 2223-2253. 
[7] M. Alava, K. Niskanen, The physics of paper, Reports on Progress in Physics 69 (3) (2006) 669.

[8] R.W. Dent, Inter-fiber distances in paper and nonwovens, Text. Inst. 92 (1) (2001) 63-74.

[9] D. Liang, B. S. Hsiao, B. Chu, Functional electrospun nanofibrous scaffolds for biomedical applications, Advanced Drug Delivery Reviews 59 (14) (2007) 1392-1412.

[10] P.A. Janmey, J. P. Winer, J. W. Weisel, Fibrin gels and their clinical and bioengineering applications, Journal of the Royal Society, Interface / the Royal Society 6 (30) (2009) 110.

[11] F.A.L. Dullien, Porous Media: Fluid Transport and Pore Structure, Academic Press (2012) 574.

[12] M.G. Kaganer, Thermal Insulation in Cryogenic Engineering, Israel Program for Scientific Translations Press, Jerusalem (1969) 75-76.

[13] J.L. Lowery, N. Datta, G.C. Rutledge, (2010). Effect of fiber diameter, pore size and seeding method on growth of human dermal fibroblasts in electrospun poly ( $\varepsilon$ caprolactone) fibrous mats. Biomaterials 31 (3) (2010) 491-504.

[14] K. Wolf, M. te Lindert, M. Krause, S. Alexander, J. te Riet, A. L. Willis, P. Friedl, Physical limits of cell migration: Control by ECM space and nuclear deformation and tuning by proteolysis and traction force, Journal of Cell Biology 201 (7) (2013) 10691084.

[15] M. Faessel, C. Delisée, F. Bos, P. Castéra, 3D Modelling of random cellulosic fibrous networks based on X-ray tomography and image analysis, Composites Science and Technology 65 (13) (2005) 1931-1940.

[16] L.T. Choong, P.Yi, G.C. Rutledge, Three-dimensional imaging of electrospun fiber mats using confocal laser scanning microscopy and digital image analysis, Journal of Materials Science and Engineering 50 (2015) 3014-3030.

[17] W. Mickel, S. Münster, L. M. Jawerth, D. A. Vader, D. A. Weitz, A. P. Sheppard, G. E. Schröder-Turk, Robust Pore Size Analysis of Filamentous Networks from ThreeDimensional Confocal Microscopy, Biophysical Journal 95 (12) (2008) 6072-6080.

[18] W.W. Sampson, Modelling Stochastic Fibrous Materials with Mathematica, Chemical Vapor Deposition, Springer-Verlag London Limited (2009).

[19] K. Schladitz, S. Peters, D. Reinel-Bitzer, A. Wiegmann, J. Ohser, Design of acoustic trim based on geometric modeling and flow simulation for non-woven, Computational Materials Science 38 (1) (2006) 56-66.

[20] K.E.Thompson, Pore-scale modeling of fluid transport in disordered fibrous materials, AIChE Journal 48 (7) (2002) 1369-1389.

[21] S.G. Advani, The Use of Tensors to Describe and Predict Fiber Orientation in Short Fiber Composites, Journal of Rheology 31 (8) (1987) 751.

[22] A. Ashari, H. Vahedi Tafreshi, A two-scale modeling of motion-induced fluid release from thin fibrous porous media, Chem. Eng. Sci. 64 (2009) 2067-2075

[23] N.K. Palakurthi, S. Konangi, U. Ghia, K. Comer, Micro-scale simulation of unidirectional capillary transport of wetting liquid through 3D fibrous porous media: Estimation of effective pore radii, International Journal of Multiphase Flow 77 (2015) 48-57.

[24] S. Jaganathan, H. Vahedi Tafreshi, B. Pourdeyhimi, Modeling liquid porosimetry in modeled and imaged 3-D fibrous microstructures, Journal of Colloid and Interface Science, 326(1) (2008) 166-175.

[25] A. Ashish Saha, S.K. Mitra, Effect of dynamic contact angle in a volume of fluid (VOF) model for a microfluidic capillary flow, J. Colloid Int. Sci. 339 (2009) 461-480.

[26] N.K. Palakurthi, S. Konangi, U. Ghia, K. Comer, Micro-scale simulation of unidirectional capillary transport of wetting liquid through 3D fibrous porous media: Estimation of effective pore radii, International Journal of Multiphase Flow 77 (2015) 48-57. 
[27] S.J. Eichhorn, W.W. Sampson, Relationships between specific surface area and pore size in electrospun polymer fibre networks. Journal of the Royal Society, Interface / the Royal Society 7 (45) (2010) 641-9.

[28] S.J. Eichhorn, W.W. Sampson, Statistical geometry of pores and statistics of porous nanofibrous assemblies, Journal of the Royal Society, Interface / the Royal Society 2 (4) (2005) 309-318.

[29] P.R. Johnston, Revisiting the most probable pore size distribution in filter media. The gamma distribution, Filtrn. and Sepn. 35 (3) (1998) 287-292.

[30] R. Bagherzadeh, S.S. Najar, M. Latifi, M.A. Tehran, L. Kong, A theoretical analysis and prediction of pore size and pore size distribution in electrospun multilayer nanofibrous materials, Journal of Biomedical Materials Research - Part A 101 A (7) (2013) 2107-2117.

[31] N.R. Lang, S. Munster, C. Metzner, P. Krauss, S. Schrmann, J. Lange, B. Fabry, Estimating the 3D pore size distribution of biopolymer networks from directionally biased data, Biophysical Journal 105 (9) (2013) 1967-1975.

[32] K. Daryabeigi, G.R. Cunnington, J. R. Knutson, Heat Transfer Modeling for Rigid HighTemperature Fibrous Insulation, Journal of Thermophysics and Heat Transfer 2013.

[33] S.-C. Lee, G.R. Cunnington, Conduction and Radiation Heat Transfer in High-Porosity Fiber Thermal Insulation, Journal of Thermophysics and Heat Transfer 14 (2) (2000) 121136.

[34] C. Bankvall, Heat Transfer in Fibrous Materials, Journal of Testing and Evaluation, 1 (3) (1973) 235-243.

[35] E.H. Kennard, Kinetic Theory of Gases, with an Introduction to Statistical Mechanics, McGraw-Hill, New York, 1938.

[36] K. Daryabeigi, G.R. Cunnington, J. R. Knutson, Measurement of Heat Transfer in Unbonded Silica Fibrous Insulation and Comparison with Theory, 29th International Thermal Conductivity Conference (ITCC) 11 (2007).

[37] K. Daryabeigi, G.R. Cunnington, J. R. Knutson, Measurement of Heat Transfer in Unbonded Silica Fibrous Insulation and Comparison with Theory, Thermal Conductivity, DEStech Publications Inc., Lancaster 29 (2008) 292-301.

[38] O.M. Alifanov, V.V. Cherepanov, Mathematical simulation of high-porosity fibrous materials and determination of their physical properties, High Temperature 47 (3) (2009) 438-447.

[39] Bozhkov, N. A., Ivanov, A. A. (1990). Conductive thermal conductivity of fibrous materials under transient gas flow. Journal of Engineering Physics, 58(5), 541-547.

[40] Cherepanov V.V. Methodology of investigation and prediction of properties of highly porous materials for thermal protection of aircrafts. Dr.Sci. Thesis, Moscow Aviation Institute, 2012.

[41] A. M. Trater, S. Alavi, S.H. Rizvi, Use of non-invasive X-ray microtomography for characterizing microstructure of extruded biopolymer foams, Food Research International 38 (6) (2005) 709-719.

[42] A. Berge, P. Johansson, Literature Review of High Performance Thermal Insulation. Report in Building Physics, Chalmers University of Technology (2012).

[43] Spinnler, M., Winter, E. R. F., Viskanta, R., Sattelmayer, T. (2004). Theoretical studies of high-temperature multilayer thermal insulations using radiation scaling. Journal of Quantitative Spectroscopy and Radiative Transfer, 84(4), 477-491.

[44] Ru, J., Zuotai, Z., Lili, L., Xidong, W. (2014). Numerical modeling and experimental study of heat transfer in ceramic fiberboard. Textile Research Journal, 84(4), 411-421.

[45] Karamanos, A., Papadopoulos, A., Anastasellos, D. (2004). Heat transfer phenomena in fibrous insulating materials. Proceedings of 2004 WSEAS/IASME International Conference on Heat and Mass Transfer, 7. 
[46] Zuev, A. V., Prosuntsov, P. V. (2014). Model of the Structure of Fibrous Heat-Insulating Materials for Analyzing Combined Heat Transfer Processes. Journal of Engineering Physics and Thermophysics, 87(6), 1374-1385.

[47] L.A. Dombrovsky, D. Baillis, Thermal radiation in disperse systems: an engineering approach. New York : Begell House (2010).

[48] M.A. Tahir, H.V. Tafreshi, S. A.Hosseini, B. Pourdeyhimi, Modeling the role of microstructural parameters in radiative heat transfer through disordered fibrous media, International Journal of Heat and Mass Transfer 53 (21-22) (2010) 4629-4637. 